\title{
EL DESARROLLO TURÍSTICO Y LA UTOPÍA DE LA SUSTENTABILIDAD EN BAHIA DE BANDERAS, NAYARIT
}

\author{
Sandra Luz Zepeda Hernández* \\ Fabíola Cristina Costa de Carvalho**
}

\begin{abstract}
El enfoque económico del turismo ha sido rebasado paulatinamente a través de estudios más complejos de la realidad que se modifica a causa de este fenómeno social. Es así como en el presente documento se plantea como concepto central el turismo como modelo de desarrollo en el sistema capitalista, en este caso, implantado a partir de la aplicación de la política pública federal. Se busca ejemplificar a través de la focalización en dos casos emblemáticos del municipio costero Bahía de Banderas, Nayarit/México, el desequilibrio social y ambiental que ha traído consigo el desarrollo del turismo masivo de sol y playa fomentado décadas atrás. Lo anterior se coloca a la luz de los objetivos planteados por la Organización Mundial del Turismo (OMT) al declarar el 2017 como el año Internacional del Turismo Sostenible para el Desarrollo. El análisis documental y la evidencia empírica permiten inferir importantes desequilibrios que intervienen en la realidad social minimizando las posibilidades de alcanzar los objetivos de la agenda OMT al año 2030.
\end{abstract}

Palabras Clave: Turismo. Sustentabilidad. Bahía de Banderas.

\section{DESENVOLVIMENTO TURÍSTICO E UTOPIA DE SUSTENTABILIDADE NA BAHIA DE BANDERAS, NAYARIT}

O enfoque econômico do turismo foi gradualmente superado através de estudos mais complexos da realidade que é modificada por causa desse fenômeno social. Assim, neste documento, o turismo é considerado como um conceito central, como modelo de desenvolvimento no sistema capitalista, neste caso, implementado a partir da aplicação da política pública federal. Busca-se exemplificar através do foco em dois casos emblemáticos do município costeiro Bahía de Banderas, Nayarit/México, o desequilibrio social e ambiental que trouxe consigo o desenvolvimento do turismo massivo de sol e praia, promovido décadas atrás. 0 que precede é colocado à luz dos objetivos estabelecidos pela Organização Mundial do Turismo (OMT) ao declarar 2017 como o Ano Internacional do Turismo Sustentável para o Desenvolvimento. A análise documental e a evidência empírica permitem inferir desequilibrios importantes que intervêm na realidade social minimizando as possibilidades de alcançar os objetivos da agenda da OMT até 0 ano de 2030.

Palavras Chave: Turismo. Sustentabilidade. Baía de Banderas.

\section{TOURIST DEVELOPMENT AND THE UTOPIA OF SUSTAINABILITY IN BAHIA DE BANDERAS, NAYARIT}

\begin{abstract}
The economic focus of tourism has been gradually overcome through more complex studies of the reality that is modified because of this social phenomenon. Thus, in this document, tourism is considered as a central concept as a development model in the capitalist system, in this case, implemented from the application of federal public policy. It seeks to exemplify through the focus on two emblematic cases of the coastal municipality of Bahía de Banderas, Nayarit/Mexico, the social and environmental imbalance that has brought with it the development of massive sun and beach tourism fostered decades ago. The above is placed in light of the objectives set by the United Nations World Tourism Organization (UNWTO) to declare 2017 as the International Year of Sustainable Tourism for Development. The documental analysis and the empirical evidence allow to infer important imbalances that intervene in the social reality minimizing the possibilities of reaching the objectives of the UNWTO agenda by the year 2030.
\end{abstract}

Keywords: Tourism. Sustainability. Bahía de Banderas.

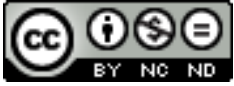

Licenciada por Creative Commons Atribuição Não Comercial / Sem Derivações/ 4.0 / Internacional

\footnotetext{
* Doctorando en Ciencias para el Desarrollo, la Sustentabilidad y el Turismo, Universidad de Guadalajara. Maestría en Dirección de Mercadotecnia, Centro Universitario de Ciencias Económico-Administrativas, U de G. Coordinador Area Análisis e Inteligencia Turística del Observatorio Integral de la Región Turística Puerto Vallarta-Bahía de Banderas. Profesor Investigador de Tiempo Completo, miembro del Cuerpo Académico Desarrollo Sustentable y Nuevas Tendencias del Turismo, Universidad Tecnológica de Bahía de Banderas. www.observatorioit.org lzepeda@utbb.edu.mx

** Doutoranda em Ciências Sociais, Universidad Autónoma de Sinaloa. Mestre em Administração Pública. Bacharel em Turismo. Integrante Centro de Excelência Latino Americano em Turismologia/CELAT e Observatório Econômico e Social do Turismo/OEST, vinculados a Universidade Federal de Juiz de Fora/Brasil. Interessa-se e atua nas áreas de Turismo, Ciências Sociais, Ciência Política e Administração Pública. [ fabiolacarvalho.tur@hotmail.com ]
} 


\section{INTRODUCCIÓN}

El desarrollo turístico en Bahía de Banderas, Nayarit/México, ha representado para el municipio una transformación de las actividades económicas, sociales y medio ambientales que se acentúa con la apertura a los grandes capitales a partir del modelo neoliberal, aquellos que se trasladan desde áreas distantes a los espacios turísticos que construyen, obedeciendo siempre la lógica del mercado y en muchos casos provocan altos costos sociales asumidos por la comunidad receptora que se convierte en una sociedad controlada por desarrolladores y empresarios turísticos (CÉSAR y ARNAIZ, 2006, CÉSAR, 2015).

En ese contexto, recientemente se ha propiciado la construcción de megaproyectos turísticos en la Riviera Nayarit y, más específicamente, en el municipio objeto de estudio. En estos territorios los grandes capitales han generado una expectativa en la generación de empleo con la venia de los gobiernos locales, estatales y federales en turno.

Por ello, inicialmente se plantea a través del presente documento una introducción a la incipiente experiencia de dos megaproyectos turísticos en curso en Bahía de Banderas. De ahí que no se pretende enunciar de forma exhaustiva el tema, por el contrario, el objetivo es una primera aproximación a los impactos de éstos en el ámbito social y ambiental al contrastarse con las metas planteadas por la OMT en miras a su cumplimiento para el año 2030.

Así, el presente trabajo toma como premisa que el turismo es una forma o modelo de desarrollo que adopta el Estado y se ejerce a través de las políticas públicas establecidas y puestas en marcha, en el marco del sistema capitalista hegemónico, partiendo de César y Arnaiz $(2006,2012)$ y César (2015) quienes plantean una visión del turismo como modelo de desarrollo inherente al sistema capitalista mismo que se despliega en contradicciones. Una de ellas, refieren los autores, es presentar el turismo como un producto maquillado de la realidad que se vende como la realidad misma, haciendo de lo transformado una nueva verdad, cuya principal función es la de responder a los imaginarios que tiene el hombre en su perspectiva de la búsqueda incesante de nuevos objetos de consumo, en este caso de ocio y placer retomando el enfoque de MacCannell (2003).

El articulo presenta una estructura de seis apartados además de esta introducción. En el segundo apartado se presenta brevemente los antecedentes del tema de la sustentabilidad y la sustentabilidad del turismo en la agenda internacional. En la tercera parte se presentan los contextos estudiados, iniciando con la contextualización del turismo en México y luego pasando a las características de las intervenciones turísticas en Bahía de Banderas, locus del estudio'. En el cuarto apartado se presenta una síntesis de los resultados encontrados. Se ha buscado lo anterior a partir de una investigación desde un enfoque integral y perspectiva interdisciplinaria (WALLERSTEIN, 2013), que permite la aprehensión de una realidad de naturaleza compleja derivado de la multiplicidad de componentes que lo forman y la interrelación que se establece entre los mismos como lo es el fenómeno turístico (VERA et al., 2013). Finalmente las consideraciones del estudio son exteriorizadas.

\section{EL TEMA DE LA SUSTENTABILIDAD EN LA AGENDA PÚBLICA INTERNACIONAL}

El concepto de desarrollo sostenible ha sido generalizado y adaptado por muchos países como la única manera de desarrollo equilibrado y justo (LINARES y GARRIDO, 2014). De acuerdo con Lopez y Godoi (2014) desde los siglos XVIII y XX, el capitalismo industrial llevó al desarrollo de las sociedades basado en explotación de los recursos humanos y naturales. Todavía la Revolución Industrial resultó en el desarrollo económico de pocos países y la exploración de los demás. En dicha circunstancias se consolidó el modelo económico capitalista, con el objetivo principal de la generación de renta a través de la explotación de los recursos disponibles.

En el escenario político internacional la crisis ambiental empezó a manifestarse en los años 1970 (CHÁVEZ y OSORIO, 2006). Resultado de la crisis provocada por las reservas de petróleo, que hasta entonces era considerado como un recurso inagotable, comenzó el debate sobre los límites al desarrollo industrial y económico (LOPEZ y GODOI, 2014).

Los debates internacionales empezaron a tratar la necesaria articulación entre el crecimiento económico y la conservación del medio ambiente, en especial respecto de los casos debatidos acerca de las relaciones entre países desarrollados y en desarrollo, sumándose, pues, la dimensión social y

\footnotetext{
${ }^{1}$ Metodológicamente este estudio fundamentalmente se basa en una reflexión teórica originada a partir de la realidad y confrontada con datos empíricos, de forma ilustrativa. Para la construcción del marco referencial y conocer los aspectos históricos, contextuales y normativos relacionados al tema, se reunió y seleccionó información a través de una investigación documental que permitió estudiar el fenómeno principalmente mediante consulta de documentos institucionales de carácter público e internet. En una segunda parte, se recolectó información de fuentes primarias, utilizando técnicas de investigación de corte cualitativo a través de la observación y recorridos en sitio, así como entrevista a profundidad (RUIZ e ISPIZÚA, 1989) de tipo semiestructurada y focalizada.
} 
ambiental al concepto y a los intentos del progreso (LINARES y GARRIDO, 2014). Es decir, el tema de la vinculación de los conceptos de desarrollo y ambiente surge en la agenda internacional en un contexto donde emergió la idea de que los costos ambientales y sociales son resultado del modo de producción y de distribución capitalista.

En 1972 se llevó a cabo la Conferencia sobre el Medio Ambiente Humano, organizada por la Organización de las Naciones Unidas (ONU) en Estocolmo, Suecia (SALVATIERRA, 2011). De este evento resultó la creación del Programa de las Naciones Unidas para el Medio Ambiente (PNUMA), con el fin de concientizar sobre la acción de los ambientalistas acerca del cuidado ambiental (LOPEZ y GODOI, 2014).

El primer concepto de desarrollo sostenible fue elaborado en 1980, por la Unión Internacional para la Conservación de la Naturaleza (UICN), el Fondo Mundial para la Vida Salvaje (WWF) y el Programa de las Naciones Unidas para el Medio Ambiente (PNUMA) (LOPEZ y GODOI, 2014). Todavía la discusión respecto a este tema se mantuvo en el ámbito académico hasta 1987, cuando la Comisión Mundial para el Desarrollo y el Medio Ambiente, publicó el documento "Nuestro Futuro Común", conocido como Informe de Brundland (CHÁVEZ y OSORIO, 2006).

La Comisión Brundland escudó por la definición de una estrategia de desarrollo sustentable (SALVATIERRA, 2011) bajo el argumento de que el desarrollo se alcanza cuando se satisfacen las necesidades fundamentales de una sociedad, como la educación, las necesidades culturales, espirituales, además de los logros en la economía (CHÁVEZ y OSORIO, 2006).

Es importante señalar que la conciencia ambiental ha aumentado significativamente en Europa posterior al accidente nuclear de Chernobyl en 1986. El concepto de desarrollo sostenible se difundió, sin embargo, en 1992 cuando se celebró la Conferencia de las Naciones Unidas sobre el Medio Ambiente y Desarrollo, en Rio de Janeiro, Brasil (conocida como Cumbre de la Tierra o ECO-92). En este evento se evaluó como los criterios ambientales fueron incorporados por los gobiernos desde la Conferencia de Estocolmo. Además, se aprobó dos convenciones sobre biodiversidad y cambio climático, que se tradujo en la elaboración del Protocolo de Kyoto en 1997 (MUNTEAL y SIMONET, 2012).

En concreto, los acuerdos se alcanzaron en la preservación del medio ambiente mediante la reducción de las emisiones de contaminantes en la atmósfera. De acuerdo con Lopez y Godoi (2014) el resultado de la ECO-92 fue el lanzamiento de los documentos oficiales:

1) Carta de la Tierra, donde se planteaba que los acuerdos internacionales establecidos deberían respetar los intereses de todos los involucrados y proteger la integridad del sistema global de ecología y de desarrollo;

2) Agenda 21, con el objetivo de implementar programas y acciones efectivas para desacelerar y detener el proceso de degradación ambiental, y de igual forma suscitar el desarrollo sostenible.

Al mismo tiempo, para promover la implantación de los acuerdos deliberados en la Conferencia y buscar la formación de consejos nacionales de desarrollo sustentable, fue creado el Consejo de la Tierra ${ }^{2}$. Después de la realización del evento algunos documentos internacionales fueron desarrollando el concepto de sustentabilidad y celebrando pautas para su aplicación, su incorporación a los espacios de discusión política y en la elaboración de las políticas públicas (CHÁVEZ y OSORIO, 2006).

Veinte años después de la consecución de la ECO-92, en 2012, también en Río de Janeiro, se llevó a cabo la Conferencia de las Naciones Unidas sobre el Desarrollo Sustentable (RIO+20), con el objetivo de renovar los compromisos firmados en la ECO-92, respecto al desarrollo sustentable en nivel global; además de identificar y evaluar las fallas de los acuerdos internacionales y de discutir otras formas de recuperar los daños ambientales mientras se sigue el progreso (LOPEZ y GODOI, 2014).

En ese contexto uno de los temas centrales de las discusiones fue la búsqueda por un punto de equilibrio entre los objetivos económicos globales y el desarrollo sustentable (OMT, 2013a).

\section{EL DESARROLLO SOSTENIBLE EN LA AGENDA DEL TURISMO INTERNACIONAL}

En el contexto de emergencia de los debates sobre el desarrollo sostenible, en la década de 1970, surgieron las preocupaciones sobre el desarrollo equilibrado del turismo y la calidad de la actividad turística a través de la conservación de los recursos naturales, sociales y culturales de los destinos turísticos (OMT, 2001; LOPEZ y GODOI, 2014).

Todavía el concepto "turismo sustentable" fue elaborado al final de la década de 1980, oponiéndose al concepto de "turismo de masa". En ese periodo hubo un aumento en los debates acerca de los

\footnotetext{
2 Más informaciones en

<http://www.cartadaterrabrasil.org/prthistory.html>. Aceso en 05 mar. 2014.
} 
impactos del turismo destructivo y desordenado, causado por la falta de planeamiento, y que resultó en pocos beneficios sociales y económicos para las comunidades receptoras, por lo demás de tener producido expresivos resultados negativos sobre el medio ambiente (CAVALCANTI, 2006).

Los temas relacionados a la sustentabilidad entran paulatinamente en las discusiones de la agenda del turismo. Aún la sustentabilidad ambiental es el que predomina en las primeras discusiones de la agenda internacional del turismo. En este contexto la Conferencia de Manila (1980) refleja la configuración de un cuadro vinculado a la consciencia ambiental en el turismo, pues en aquel momento se destacó el debate que resultó en la adopción de nuevos límites al desarrollo de la actividad turística (LOPEZ y GODOI, 2014).

Después de ese evento la Organización Mundial de Turismo (OMT) organizó reuniones y encuentros para debatir ese tema. De acuerdo con Lopez y Godoi (2014) los resultados de dichas discusiones fueron varios documentos oficiales como declaraciones de intenciones, códigos de conducta y reglamentaciones para la actividad turística ${ }^{3}$.

En 1991, la Asociación Internacional de Expertos Científicos en Turismo (AIEST, en sus siglas en inglés), conceptuó que el turismo sustentable como aquel que mantiene el equilibrio de los intereses sociales, ecológicos y económicos, además de conservar los valores los naturales y culturales de una localidad (LOPEZ y GODOI, 2014). En este momento ya se demuestra inserido en el discurso institucional otros ejes, además del medio ambiental, como temas relacionados a la sostenibilidad del turismo.

En 1993 la OMT definió que el turismo sustentable atiende a las necesidades de los turistas y de los destinos turísticos, mientras protege y amplía las oportunidades para el futuro, valorando la cultura nacional (Lopez y Godoi, 2014). Luego la "Carta de Turismo Sustentable de Lanzarote" (1995) señala que el desarrollo sustentable es un proceso orientado de gestión de los recursos naturales y culturales en nivel global, con la finalidad de la conservación a las generaciones futuras.

Notase la inserción de la Organización Mundial de Turismo (OMT) y del World Travel and Tourism Council (WTTC) en una posición central en los debates internacionales sobre la sostenibilidad en el turismo. Así en 1996, entre otros documentos producidos, estos actores presentaron al Consejo de

\footnotetext{
${ }^{3}$ Algunos documentos elaborados en la década de 1980 son la "Carta del Turismo" y el "Código del Turista" en 1985, la "Declaración de Tamanrasset" en 1989, la "Declaración de Haia sobre Turismo", en 1989. (cf. Lopez y Godoi, 2014).
}

la Tierra la "Agenda 21 para el sector de los viajes y el turismo", recomendando la necesidad de formar alianzas entre los sectores público y privado, además de la sociedad civil que participa en las actividades de la industria del turismo (RABINOVICI, 2011).

En ese periodo los mecanismos para fomentar la participación de los involucrados en la cadena productiva del turismo y otros actores sociales interesados en el desarrollo turístico se volvieron una de las bases para la elaboración y la implementación de políticas públicas de turismo. Así que se direccionó las orientaciones y recomendaciones relacionadas a la desreglamentación y la gestión descentralizada del sector, difundidas a partir de la década de 1980 en Europa y Estados Unidos y de los 1990 en América Latina.

A su vez, en las dos primeras décadas del siglo $X X I$, el discurso político vincula el concepto de sostenibilidad a una posibilidad para el desarrollo social. En este período se llevó a cabo dos eventos importantes en el año internacional del ecoturismo (2002): en primero lugar la Cumbre Mundial del Ecoturismo, en Québec, Canadá; ocasión en la que se reconocía que la Cumbre Mundial sobre el Desarrollo Sostenible (Rio+10), que iba a ser celebrada en Johannesburgo, África, de aquel mismo año, se sentarían las bases de una política internacional para los próximos diez años. En la misma conferencia se reconoció que la sostenibilidad del turismo debe ser una prioridad, por su potencial de contribuir para reducir la pobreza y proteger el medio ambiente en ecosistemas amenazados.

En 2010 la Organización Mundial del Turismo formó el "Global Sustainable Tourism Council" como una organización dedicada a promover la adopción de los "Criterios Globales de Turismo Sostenible" y promover las buenas prácticas ambientales concernidas con la industria del turismo mundial (SECRETARÍA DE TURISMO DE MÉXICO, s/d).

Posteriormente, en la Conferencia Rio +20 , en 2012, el turismo fue incorporado por primera vez a un documento de la ONU acerca de los resultados sobre sustentabilidad, cuando se reconoció que él sector es capaz de contribuir para la sustentabilidad económica, social y ambiental, pues está fuertemente vinculado a otros sectores de la economía, contribuido especialmente para la generación de empleos y consecuentemente, la reducción de desigualdades sociales (OMT, 2013).

El 2017 fue declarado en la Asamblea General de las Naciones Unidas del 2015 como el año Internacional del Turismo Sustentable para el Desarrollo (UNWTO y UNDP, 2017). Así se ha establecido 17 objetivos para transformar el mundo, mismos que han sido suscritos por los miembros de la 
OMT incluyendo a México, los cuales deben de ser considerados en las agendas políticas hasta el 2030.

En este contexto para que sea posible la concretización de la agenda del turismo sustentable propuesta por la OMT es necesario utilizar el concepto de sustentabilidad del turismo que utiliza una perspectiva más compleja; pasando a caracterizarse por el desarrollo de una interferencia organizada y planeada, para que se reduzcan los problemas generados en los destinos turísticos (SOARES y EMMENDOERFER, 2011).

\section{EL DESAROLLO DEL TURISMO EN MÉXICO}

Diversos estudios sobre el desarrollo del turismo en México consignan que éste representó una salida hacia el desarrollo regional en zonas deprimidas del país. Históricamente, el puerto de Acapulco sirvió como puente comercial Filipinas-Nueva España, aunque su fama internacional se debió mucho tiempo después a factores externos en las primeras décadas del siglo XX relacionados con la economía capitalista estadounidense, entre ellos el desarrollo de la industria automotriz, cuya presión por la construcción de carreteras para dar salida a sus productos rindió efecto en México, conectando a la ciudad de Acapulco con la capital lo que facilitó la llegada de visitantes acompañados por empresarios del sector (GÓMEZ, 1974).

De manera análoga, Puerto Vallarta en la costa jalisciense, desconocido hasta la década de los cincuenta, salta a la fama internacional en 1963 derivado del rodaje del filme La Noche de la Iguana y, principalmente, por el romance entre dos personajes del jet set hollywoodense, Richard Burton y Elizabeth Taylor. Es así que para inicios de la década de los sesenta se contaba con un destino consolidado y otro incipiente en el Pacífico; el entramado de intereses políticos y económicos, enmarcado en el modelo de desarrollo nacional, abre la puerta para la inversión público-privada en forma de política pública que llevará a la creación de Centros Integralmente Planeados (CIP) en el litoral de los Estados de Quintana Roo, Guerrero, Oaxaca, Baja California Sur y, recientemente, en los 2000, en Nayarit y Sinaloa.

Así a fines de la década de 70 el gobierno y la iniciativa privada impulsaron el turismo masivo, en Puerto Vallarta por medio de importantes inversiones para la construcción de infraestructura, que transformaron el panorama local (GUARDADO, 2009).

La proximidad del municipio de Bahía de Banderas con Puerto Vallarta derivó en una mirada hacia el turismo como alternativa a los problemas que enfrentaba la entidad aprovechando el entorno nacional e internacional. A nivel federal, en 1970 mediante un decreto presidencial, Gustavo Díaz Ordaz ordenó la expropiación de terrenos costeros con el fin de destinarlos a desarrollos en 8 ejidos del entonces municipio de Compostela. En este mismo año, en la administración federal de Luis Echeverría Álvarez se crea el Fideicomiso de Bahía de Banderas (FIBBA) con la encomienda de administrar los predios expropiados y lograr el desarrollo de los terrenos habitacionales y turísticos expropiados, definiendo a la misma como una alternativa confiable para la inversión turística del Estado y promover el desarrollo regional (FIBBA, 2017).

Se generó entonces una política estatal durante 1988-1993 que implicó el desarrollo económico como componente básico orientando los esfuerzos hacia la diversificación de la estructura económica y el equilibrio regional considerando al turismo. Es en este período que se enfatiza el aspecto regional como elemento de desarrollo y el turismo como alternativa para la promoción del desarrollo de Nayarit (GOBIERNO DEL ESTADO DE NAYARIT, 1988; CASTRO, 2010), hechos coincidentes con la separación territorial de Compostela, formalizando la creación del municipio número veinte del estado de Nayarit en diciembre de 1989, mediante el decreto 7261 en cuya legalidad queda asentado la creación de Bahía de Banderas como municipio libre (INAFED, 2017).

La década de los noventa se distingue por las inversiones dirigidas hacia el municipio de Bahía de Banderas, lo que resultó a nuevas zonas de desarrollo y la renovación del interés público por apoyar el turismo de gran envergadura y altamente competitivo internacionalmente, como los proyectos Costa Banderas y Punta de Mita, que sin embargo han resultado, en centros turísticos muy exclusivos "que no siempre acarrearon beneficios a las sociedades locales" (GUARDADO, 2009, p. 223).

Para el siglo XXI, la política turística federal contenida en el Programa Nacional de Turismo 20012006 se articuló con base en cuatro ejes, de los cuales derivan objetivos sectoriales, destacando el que enuncia al turismo como prioridad nacional, que a su vez establece como objetivo el diseño e impulso a una política de Estado en materia turística.

En congruencia con lo anterior, durante el período del gobernador nayarita Ney González Sánchez se asume a la actividad turística como una prioridad y elabora el Programa Estatal de Desarrollo Turístico 2008-2011 el cual establece tres ejes rectores: Sustentabilidad, Competitividad y Diversificación. De este último se desprende la estrategia "Macro proyectos de los sectores productivos estratégicos", cuyas líneas de acción son la elaboración de un plan de mercadotecnia para dotar a Nayarit de una marca 
propia como destino turístico, concretándose así el registro de marca Riviera Nayarit ante el Instituto Mexicano de Propiedad Industrial (IMPI) en el 2007 y posteriormente las denominaciones Lagunas Encantadas, Nayarit Colonial y Sierra del Nayar.

\subsection{Bahía de Banderas y los Megaproyectos: casos significativos}

En estricta definición, megaproyecto, puede ser definido de acuerdo a la Real Academia Española como el "designio o pensamiento de ejecutar una obra de arquitectura o ingeniería de grandes dimensiones", en congruencia con Díaz Orueta (2009) quien distingue en el término "megaproyecto urbano" dos acepciones en función del tipo de intervención en cuestión?

La primera, refiere a las intervenciones articuladas alrededor de la construcción de un gran edificio dotado de una fuerte carga simbólica (como puede ser un museo); la segunda, tiene las intervenciones más amplias y con un contenido complejo donde se pueden mezclar usos residenciales y de actividad terciaria, en las que se utilizan nuevas técnicas financieras que fortalecen una estrecha colaboración entre el sector público y el privado (LEHRER y LAIDLEY, 2006; DÍAZ, 2009, p.194).

Con base en lo anterior, es posible considerar como megaproyectos a dos proyecciones turísticas que por sus dimensiones y naturaleza pueden ser un referente en Bahía de Banderas. Por un lado, Litibú, la primera fase del CIP Nayarit, y más recientemente, la puesta en marcha de la construcción del complejo Vidanta, ubicado en la zona limítrofe entre Jalisco y Nayarit.

Figura 1: Ubicación Litibú y Vidanta.

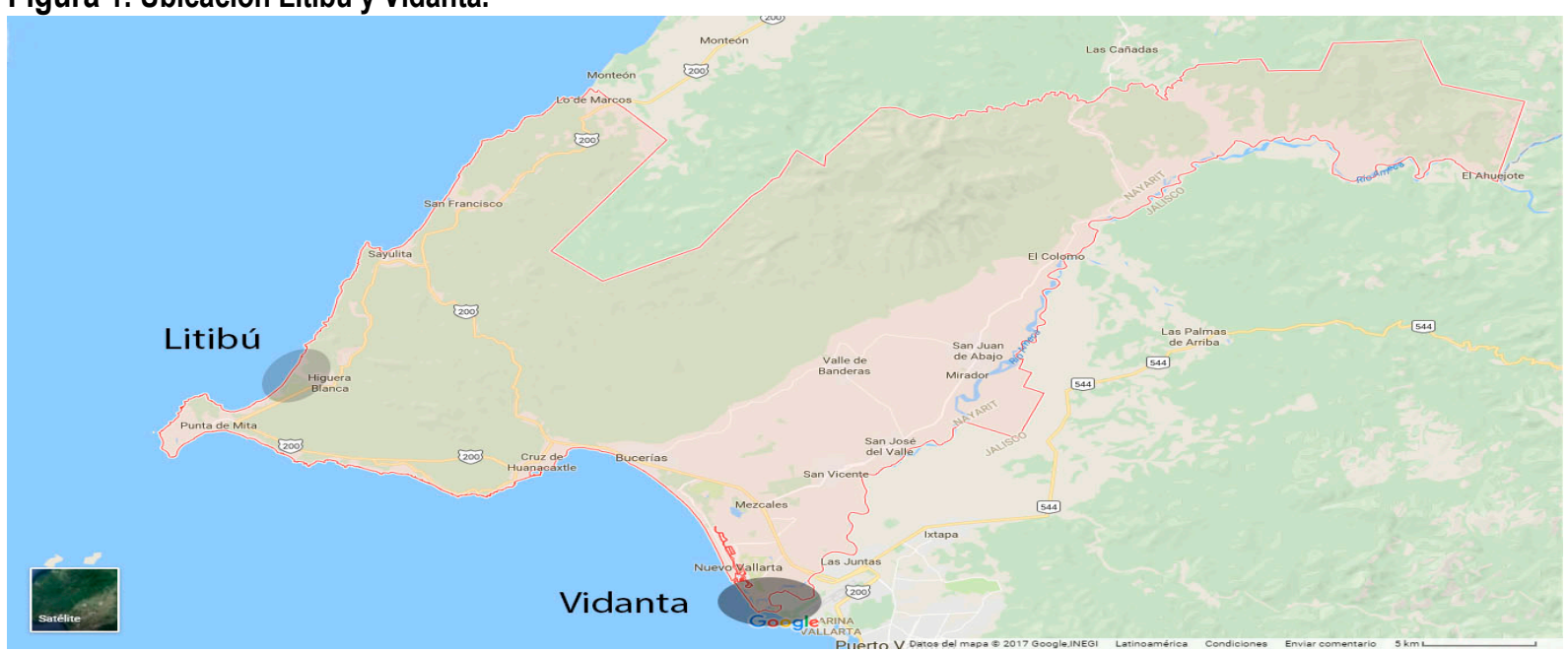

Fuente: Google Maps, 2017.

\subsection{Litibú, Breve Historia de un CIP Inconcluso}

Como parte de la política turística en México, durante el año 2003 se adquirieron los terrenos por el Fondo Nacional de Fomento al Turismo (FONATUR) al Fideicomiso de Bahía de Banderas (FIBBA), iniciando con ello Litibú, la primera parte del proyecto CIP Nayarit, en un polígono correspondiente a 151.84 ha el cual se ubica a $3 \mathrm{Km}$ al norte de Punta Mita; la segunda y tercera etapas corresponden a los polígonos El Capomo (hoy denominado Costa Canuva) y La Peñita de Jaltemba en el municipio de Compostela, aunque es de señalarse que en el caso último las negociaciones no se concretaron para la adquisición de los terrenos ejidales, empero, el proyecto tuvo inicialmente una proyección a finalizar en el año $2025^{4}$.

\footnotetext{
3 "Mega", del griego mega, al igual que "macro" del griego makro, significa "grande" y; "proyecto", del latín proiectus, que significa en una de sus acepciones la "ejecución de algo de importancia, designio o
}

Cabe señalar que, en 2016 Costa Canuva fue adquirido por Mota-Engil convirtiéndose en la mayor inversión turística extranjera para México, este desarrollo turístico también contará con un campo de golf diseñado por Greg Norman y la golfista mexicana Lorena Ochoa (FONATUR, 2017).

Originalmente el proyecto Litibú incluye la creación de 4,100 habitaciones, campo de golf 18 hoyos, centro comercial y de entretenimiento y clubes de playa. De esta forma, se enfocaría al turismo de sol y playa en el segmento de altos ingresos, con la

pensamiento de ejecutar, o bien, el conjunto de escritos, cálculos o dibujos que se hacen para dar idea de como ha de ser y lo que ha de costar una obra de arquitectura o ingeniería" (RAE, 2017); en este sentido estricto, se puede tomar "macroproyecto" y "megaproyecto" como sinónimos.

${ }^{4}$ La vigencia de las acciones queda sujeta a las adminstraciones públicas federales, aspecto que ha influido en la toma de decisiones para la concreción de objetivos, en este caso se toma como referencia El Libro Blanco y los proyectos ejecutivos disponibles a través de Transparencia FONATUR. 
salvedad de la diferenciación de hospedaje entre extensiva-intensiva como ajuste al modelo en función de las preferencias de ese segmento. Sin embargo, se observa que la baja densidad y el menor impacto ambiental se superponen igualmente, debido a la deforestación que "fue necesaria" para la implantación del proyecto, así como el mantenimiento permanente con agroquímicos del campo de golf, plus de los servicios que se ofertan (FONSECA MORALES, 2009, p.99).

"Litibú representa hasta hoy una inversión total por parte del Gobierno Federal de alrededor de 750 millones de pesos, que se suman a una inversión privada estimada en cinco mil millones de pesos en las diferentes fases del proyecto; esto permitirá generar tres mil empleos directos y casi 10 mil empleos indirectos" (Felipe Calderón, Presidente de México 2006-2012).
Figura 2: Entrada principal Litibú.

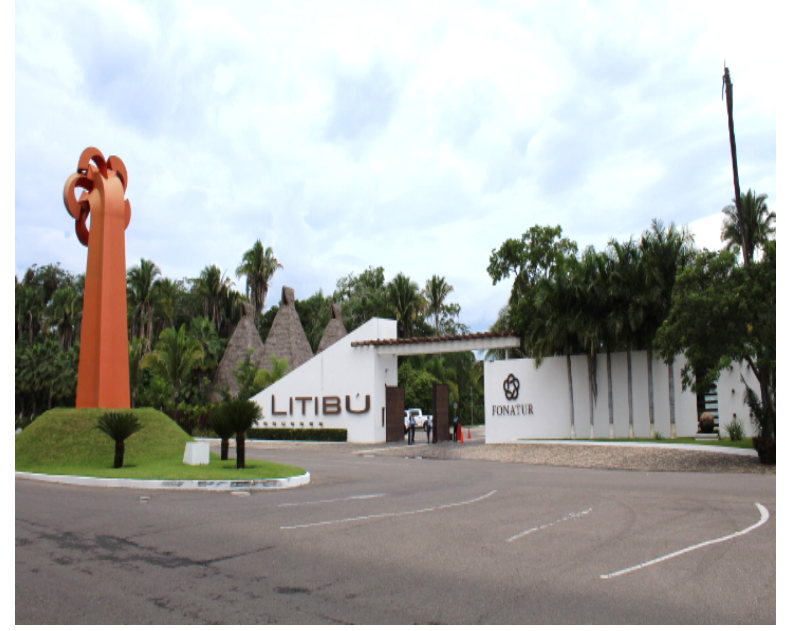

Fuente: Propia, 2017.

Tabla 1. Programa de Desarrollo CIP Nayarit.

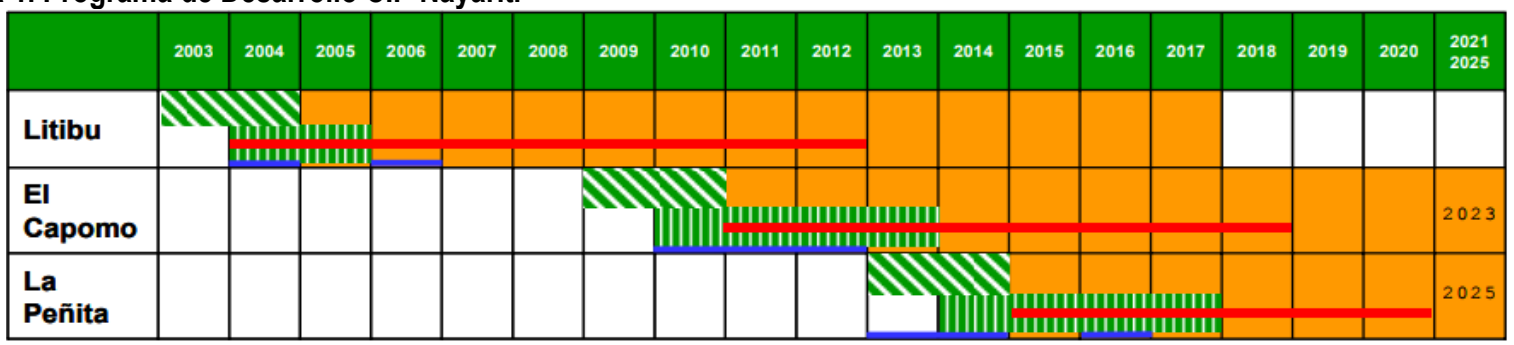

\section{MN 1. Estudios y proyectos}

Fuente: Fonatur, 2006.

\section{Campo de Golf}

Asimismo, de acuerdo al Programa de Desarrollo del CIP (Ver tabla 1), se tiene proyección de recuperación de créditos al 2017, lo que permite inferir un resultado adverso con base en el registro actual de las inversiones en Litibú, mismas que corresponden a los desarrollos La Tranquila e Iberostar y cuyo número de cuartos disponibles asciende a un aproximado de 508 habitaciones $^{5}$ en su conjunto, muy inferior a las cifras esperadas para el presente año.

De acuerdo a declaraciones del entonces Presidente de México, Felipe Calderón Hinojosa, al año 2010 se tenía una inversión aproximada de 750 millones de $\operatorname{pesos}^{6}$, y se afirma que 3 de 10 desarrolladores privados comenzaban su construcción; los restantes de acuerdo a contrato se detonarían ese mismo año.

\footnotetext{
${ }^{5}$ De acuerdo a información de la Oficina de Visitantes y Convenciones de la Riviera Nayarit. La estimación de habitaciones es la suma de cuartos, suites y residencias disponibles de ambos complejos.

6 Boletín prensa No. 05/10 de FONATUR.
}

Periodo de recuperación de créditos.

\subsection{Litibú y el Desarrollo Sustentable}

Adicional a las notables diferencias identificadas en la ejecución y recuperación económica del megaproyecto, como parte del compromiso con los ejes de la sustentabilidad, Litibú contempló en su proyecto acciones de beneficio social y de bajo impacto ambiental, mismas que en la actualidad contrastan con la realidad observada. La zona de influencia directa de Litibú es la localidad de Higuera Blanca, población de 1,360 habitantes al censo del 2010 y con un grado promedio de escolaridad de 7.2 años, por debajo de la media municipal de 8.6 años. De igual forma, el $67 \%$ de los habitantes de esta localidad se encuentra con derechohabiencia a servicios de salud, porcentaje inferior al estimado municipal de $71 \%$ (INEGI, 2010).

De acuerdo a versiones, durante el período 20052008 se dio un impulso al empedrado y mejoramiento de las calles por parte de las autoridades con el objeto de contribuir a las obras de desarrollo social en la comunidad afectada, en este caso Higuera Blanca, aunque algunos de sus habitantes van más allá y mencionan que: 
“...ese desarrollo Litibú, estamos hablando que había palmeras de coco de aceite, había otro tipo de árboles que para el medio ambiente si afectó el haberlos derribado aun a pesar que plantaron otro tipo de palma más chica, pero ahi era una zona espesa que se derribaron para hacer ese desarrollo [...] aun así, el gobierno municipal nos comentaba, son los afortunados ahorita, Higuera Blanca (sic)"7

Asimismo, en torno a la forma y estilo de vida de la población autóctona se menciona que

"desde que se pobló este lugar la gente se dedicó a la pesca y así se inició su vida para sobrevivir (...) la gente sigue yendo a pescar, pero como hay más gente hay menos producto". los compromisos adquiridos por el gobierno federal. Estas opiniones encontradas se asocian principalmente con el incumplimiento del pago acordado desde la década de los 70's durante la expropiación de tierras ejidales con fines turísticos, lo que generó inconformidad por la población residente al no recibir el pago completo establecido, así como la entrega de lotes urbanizados y utilidades que esperaban como retribución por sus tierras, ya sea por expropiación directa o comunal. Por otro lado, otro de los aspectos positivos que refieren es la poca restricción que tienen para acceder a la playa, puesto que "solo ocasionalmente" hay restricción en la playa, en palabras de los pobladores "en algunas ocasiones se restringe la playa carrilleros (...) ha habido seguridad privada y militares"8
Tabla 2. Compromisos adquiridos por FONATUR.

\begin{tabular}{l} 
Acceso público a playa equipado con servicios, estacionamiento y \\
áreas comerciales. \\
\hline Camino de acceso al club de playa. \\
Saneamiento de las aguas residuales del poblado a través de su \\
conexión con la planta de tratamiento de aguas negras Litibú. \\
Mejoramiento de la calle Amado Nervo e integración de la misma \\
al camino de acceso a la playa pública. \\
\hline Mejoramiento de la zona deportiva; incorporación de malla, áreas \\
de sombra y servicios. \\
\hline Rectificación de trazo y ampliación de sección vial del Boulevard \\
que integra Higuera Blanca-Litibú-Punta Mita. \\
\hline Mejoramiento del acceso al poblado desde el Boulevard. \\
Rediseño y equipamiento de la Plaza Principal, atrio, juegos \\
infantiles y área deportiva. \\
\hline Mejoramiento y equipamiento del pozo de agua potable. \\
Fuente: Datos directos, 2017.
\end{tabular}

De acuerdo a FONATUR (2006) la obra social del megaproyecto se centró en los rubros descritos en la tabla 2, en la que se describe además la situación actual observada y relatada por pobladores. Derivado de la dificultad para proveer agua potable en la zona (por las condiciones naturales del lugar) el abasto de agua para el complejo se previó a través de la construcción de una desalinizadora que fue inaugurada por el ejecutivo federal en 2010, misma que no se encuentra en operación por los altos costos.

De igual forma pareciese haber una percepción positiva en torno al desarrollo turístico en lo general, asociado a la generación de empleo, sobre todo por las nuevas generaciones quienes se sienten satisfechas con emplearse en los complejos hoteleros, aunque opinión no compartida por aquellos pobladores nativos de edad avanzada quienes refieren la falta de cumplimiento de

\footnotetext{
${ }^{7}$ Declaraciones de José Ángel Valdivia García, Delegado Municipal de Higuera Blanca 2002-2005.

${ }^{8}$ lbid.

${ }^{9}$ La operación y el cobro de los senvicios de la red de atarieas y líneas de conducción de alcantarillado, sanitario, así como el suministro de agua potable se encuentra a cargo del organismo operador municipal, el tratamiento de las aguas residuales es operado por personal del complejo.
}

\section{Situación actual}

Solo hay un acceso de terraceria en malas condiciones

(Ver imagen 3).

No existe.

Cumplido ${ }^{10}$

\section{Cumplido}

Inconcluso. Solo se cuenta con una malla perimetral en una parte, no hay áreas de sombra ni servicios.

Cumplido

\section{Cumplido}

Inconcluso. El apoyo para mejorar la Plaza Principal lo otorgó el Gobierno del Estado.

Cumplido.

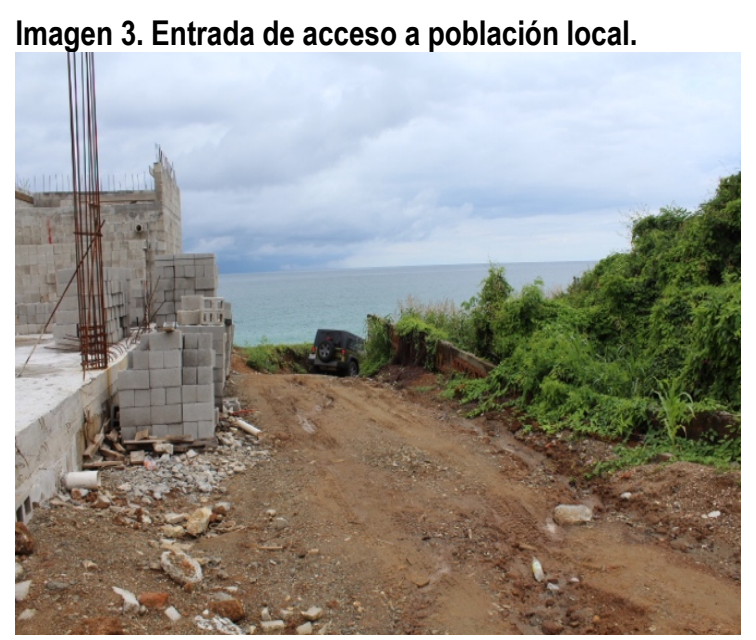

Fuente: Propia, 2017.

\subsection{Vidanta Nuevo Vallarta: un mega proyecto en curso}

Grupo Vidanta, nace en 1974 en Mazatlán, Sinaloa, convertido al 2017 en una de las mayores compañías hoteleras del segmento de lujo en destinos de playa, sus complejos tienen presencia además de 
Nuevo Vallarta, en Riviera Maya, Los Cabos, Acapulco, Puerto Vallarta, Mazatlán y Puerto Peñasco, donde también opera un aeropuerto privado. Es la mayor operadora de campos de golf, tiene 15,000 empleados y sus ingresos ascienden a 700 millones de dólares (mdd), de acuerdo al CEO del grupo empresarial ${ }^{10}$.

La concreción de un mega desarrollo de las dimensiones de Vidanta en la franja turística de Nuevo Vallarta en Bahía de Banderas, implica un esfuerzo mayúsculo en inversión y de gestión ante las autoridades competentes. Este complejo de más de 1,000 ha cuenta actualmente con 5 hoteles, $2 \mathrm{~km}$ de playa, 40 bares y restaurantes, 1 mercado gourmet, 28 albercas, 2 canchas de tenis, 2 campos de golf (Nicklaus Design y Norman Signature respectivamente) y uno más en construcción de 10 hoyos, así como una inversión de 1,300 mdd en conjunto con la empresa canadiense de entretenimiento Cirque Du Soleil para la creación de un parque temático, lo que le convierte en la mayor inversión del sector en México y por su tipo la primera en Latinoamérica.

Más aún, se anunció en 2016 la inversión adicional de 150 mdd que realizará con Hakkasan, el mayor grupo de restaurantes y clubes nocturnos del mundo, que arrancará de acuerdo a declaraciones del presidente del grupo empresarial Daniel Chávez Morán, con una serie de beach clubs, discotecas y un hotel (EXPANSIÓN, 2016).

\section{Imagen 4: Entrada principal Vidanta (sobre carretera 200, vista sur-norte).}

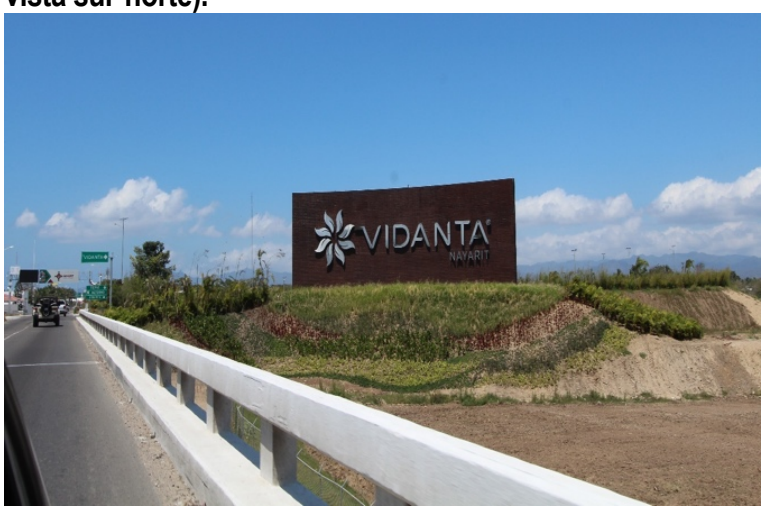

Fuente: Propia, 2016.

La zona de influencia del megaproyecto no solo es el poblado más cercano, Las Jarretaderas, mismo que ha quedado atrapado geográficamente por los desarrollos turístico-inmobiliarios a partir de la década de los 90's (Ver imagen 5), sino que actualmente va más allá de los límites político-administrativos y alcanza el vecino estado de Jalisco.

Como puede observarse en la imagen 5 , los

10 De acuerdo a Daniel Chavez, Presidente del consorcio Grupo Vidanta (CEO) en entrevista para Forbes México. puntos señalados muestran los complejos hoteleros del desarrollo, cuyos límites actualmente alcanzan los terrenos aledaños a lo largo del rio Ameca hasta llegar al poblado de Las Juntas en Puerto Vallarta, donde a través de la malla ciclónica se puede observar la maquinaria y camiones rotulados con la marca de la empresa.

\section{Imagen 5: Vidanta y su zona de influencia directa.}

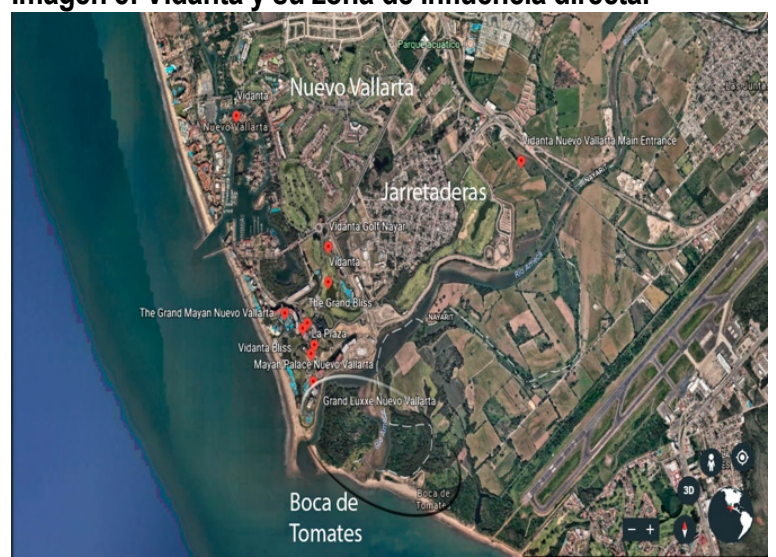

Fuente: Google maps, 2017

\subsection{Las Jarretaderas y Boca de Tomates, la Encrucijada}

La construcción del mega desarrollo ha venido acompañada de una serie de denuncias legales por las implicaciones ambientales y sociales que trae consigo la expansión del proyecto. Entre otras cosas, se le señala alterar el medio ambiente al modificar el cauce del rio Ameca y extracción de material pétreo.

El camino de acceso a documentos oficiales de la SEMARNAT que ayuden a explicar el proceso de expansión y sus posibles implicaciones en la localidad ha sido accidentado desde su inicio, medios de comunicación de circulación local, estatal y nacional han desvelado recientemente que las autoridades federales han confirmado la existencia de una concesión para el aprovechamiento de $62,545.31 \mathrm{~m}^{2}$ de terrenos ganados al mar del lugar que se conoce como Boca de Tomates, perteneciente a la delegación de Las Juntas en Puerto Vallarta, tal concesión registrada bajo el expediente 1088/JAL/2012 que concede el título DG2F/720/12 emitida por la Dirección General de Zona Federal Marítimo Terrestre y Ambientes Costeros de la Secretaría de Medio Ambiente y Recursos Naturales, otorgada a la empresa RGL Arrendadora de Inmuebles S.A. de C.V., vinculada a Grupo Vidanta $^{11}$.

11 El documento referido ha sido expuesto en medios locales después de reiterada negación por autoridades federales de socializarlo durante los últimos 4 años. 
Las Jarretaderas, con 6,262 habitantes al último censo y cuya población nacida en otra entidad ya ascendía al $72 \%$ del total (INEGI, 2010), ha presentado un elevado índice de migración de diversas entidades federativas, principalmente Chiapas y en menor proporción Guerrero, Oaxaca, Tabasco y Veracruz; estados cuyas condiciones socioeconómicas son factores de expulsión de habitantes hacia zonas del país de mayor dinámica económica donde es posible emplearse como mano de obra en la construcción en los desarrollos turísticos (CÁRDENAS, 2014; RIVERA et al., 2015).

El fenómeno migratorio implicó un aumento en la demanda de servicios públicos, tales como seguridad, transporte, salud y educación. Los efectos sociales de estos altos flujos migratorios han sido documentados previamente (CÁRDENA, 2014, 2015; RIVERA, et al., 2015), generalmente derivando en problemáticas de alcoholismo, riñas callejeras y discriminación, además de otros delitos facilitados, en cierta medida, por la falta de vigilancia por la autoridad municipal, pues se sienten "abandonados"12

A través de recorridos por el lugar, queda en evidencia el trabajo constante que maquinaria y vehículos rotulados de la empresa realizan en la zona, en congruencia con la queja reiterativa de pobladores en torno a una violación a sus derechos al dejarlos vulnerables por la remoción de material pétreo y modificando el nivel del terreno muy cerca de sus casas lo que ocasionará inundaciones de acuerdo a activistas $^{13}$.

\section{Imagen 6. Modificación al terreno en Las Jarretaderas.}

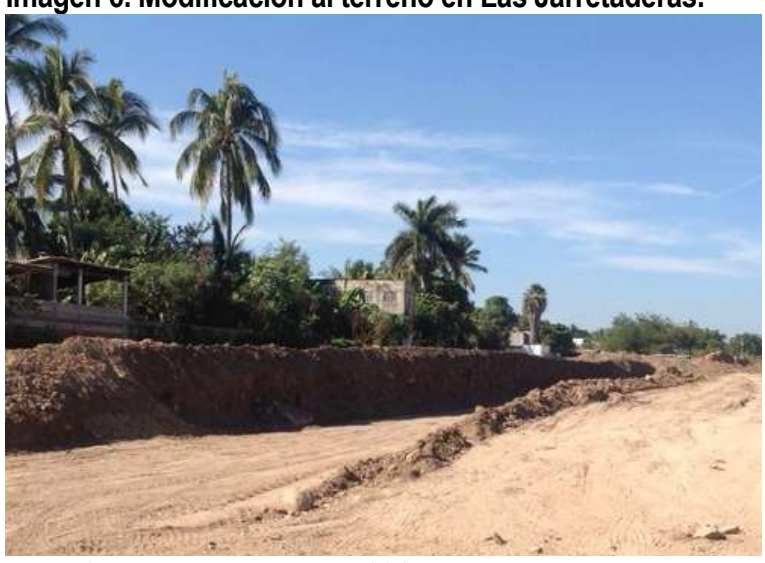

Foto: Santos, J., La Jornada; 2017.

Este hecho puede percibirse como un posible hostigamiento para que "por propia voluntad" decidan vender sus propiedades, práctica que, de acuerdo con opiniones expresadas de pobladores, es habitual para apropiarse poco a poco de los predios del pueblo con fines expansionistas.

Es así como la vista del paisaje en los alrededores del pueblo deja en evidencia su situación: por un lado, un pueblo rodeado de toneladas de construcción para abrir paso al desarrollo del turismo (Ver imagen 7) y por otro, un rio que está siendo modificado en su cauce para los mismos fines.

Imagen 7. Vista al lado norte, desde la entrada de Las Jarretaderas.

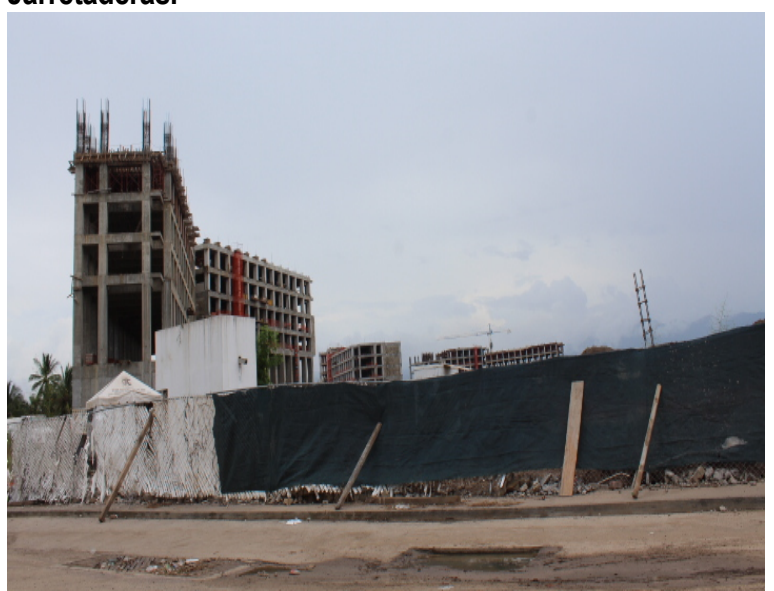

Fuente: Propia, 2017.

Parte de lo mismo es la problemática suscitada en la tradicional zona de Boca de Tomates, en Jalisco, denominada así por ser la desembocadura del rio Ameca y cuya playa ha sido el hogar de una abundante biodiversidad así como durante décadas fuente de empleo de los "palaperos", aquellos que han hecho de una ramada su negocio familiar ofreciendo servicios de alimentos (mariscos) principalmente al mercado local.

Imagen 8. Palapas en Boca de Tomates.

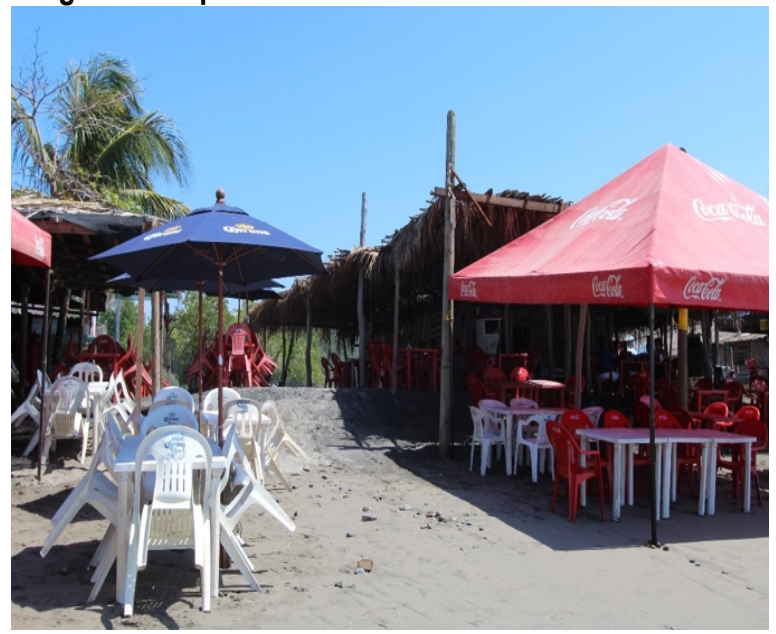

Fuente: Propia, 2016

12 De acuerdo a la percepción de habitantes oriundos de Las Jarretaderas recabada a través de entrevistas personales.

12 Declaraciones que pueden ser consultadas en http://www.jornada.unam.mx/2017/01/09/estados/027n1est 
La amenaza de desalojo se encuentra latente derivado de la concesión emitida por la SEMARNAT, como se ha confirmado por las autoridades locales y federales. Los once propietarios de las palapas temen por su fuente de ingreso, así como por la injusticia que representaría el no regresar a esa playa, "He vivido aquí todo el tiempo, aquí aprendí a caminar. Mi padre es nativo del lugar ${ }^{14}$.

En este sentido, tanto los palaperos como la población local quedan vulnerables ante la expansión inminente del desarrollo turístico, a pesar de la resistencia social por conservar su estilo de vida tradicional. Boca de Tomates ha sido un punto de acceso para pescadores desde el inicio de los pueblos costeros, por lo que también esta actividad de subsistencia familiar queda amenazada, al igual que la Isla de Los Pájaros, refugio de aves migratorias ubicado en medio del río y el estero, hábitat de cocodrilos, aves y tortugas.

\section{Imagen 9. Lanchas en Boca de Tomates.}

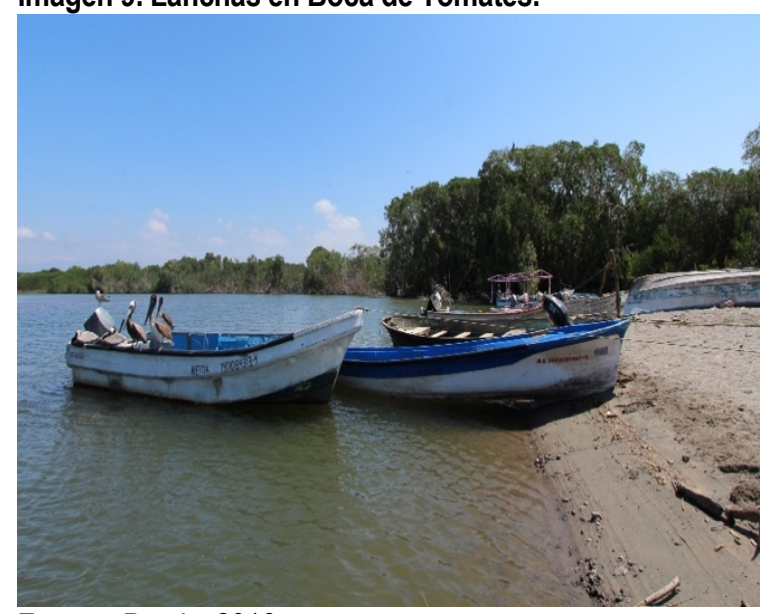

Fuente: Propia, 2016.

\subsection{Discusión}

Los dos casos presentados, de los desarrollos turísticos de Litibú y Vidanta Nuevo Vallarta, en Bahía de Banderas, han evidenciado la desconexión entre lo planteado en el discurso institucional (político) y la acción concreta del sector público y privado, cuanto a las intervenciones turísticas, su gestión sustentable, y los efectos de este contexto.

Según argumentado por Soraes y Emmendoerfer (2011), a través de la combinación entre la planeación y organización responsable es posible lograr la sustentabilidad del turismo. Así para que se cumpla la agenda OMT en los casos estudiados seria necesario que, por un lado, los gobiernos y por otro los

\footnotetext{
14 Declaración de Cecilia Espino, dueña de palapa, documentado por Santos, J., publicado en La Jornada, el 26 de marzo de 2016.
}

inversionistas y desarrolladores de los megaproyectos turísticos, cumplan efectivamente su papel y busquen en sus acciones la calidad a la experiencia de los turistas, sino también la calidad de vida a la población residente de su entorno, que son directa y continuamente afectados por el turismo.

En este contexto algunas acciones concretas que deberían verificase en un destino turístico sustentable seria la atención a las normas de intervención territorial y natural, la extensión de la infraestructura construida para el turismo a las poblaciones residentes del entorno, la oferta de oportunidades de trabajo digno a los residentes. También los impactos indirectos del turismo, como el aumento de las tasas de consumo de drogas y alcohol, además de la prostitución, que impactan en los índices de seguridad, son algunos de los problemas que deben ser identificados y tratados por las políticas públicas.

Otro problema observado es que la expansión turística atormenta el sentido de pertenencia de la gente que ha vivido en estos lugares transformados por la dinámica turística y puede generar la exclusión social, por ejemplo, por medio de los bloqueos a los accesos a las playas, sea para el ocio o para el trabajo. Sin enbargo estos son efectos no solamente del turismo, sino del modelo hegemonico (CÉSAR y ARNAIZ, 2006; CÉSAR, 2015), que para promover la prosperidad genera efectos perversos y contradictorios, y que funcionan como un motor que mantiene el propio sistema de dominación, por la creación de imaginarios, en que la esencia es consumo de lugares y experiencias.

\section{COMENTARIOS FINALES}

Los Objetivos del Desarrollo Sostenible ${ }^{15}$ buscan poner fin a la pobreza, luchar contra la desigualdad y la injusticia y hacer frente al cambio climático para el año 2030. En este sentido el establecimiento de las políticas públicas de turismo se justifican por el discurso de la sostenibilidad que se relaciona a la actividad. En el caso especifico de México la vertiente neoliberal que sigue los gobiernos ha establecido una relación cercana con las inversiones de capital privado para el desarrollo de los Centro Integralmente Planeados desde su primera generación hasta la década actual. Estos megaproyectos, sin embargo han contribuido para el desarrollo turístico del país, aunque los costos para las

15 Si bien existe un debate en relación al uso de los términos "sostenible" y "sustentable" en la literatura, para el caso que ocupa la presente investigación se utilizan de manera indistinta en virtud de la falta de distingo por la OMT y los documentos oficiales emitidos por los países miembros, entre ellos México. 
poblaciones cercanas a los emprendimientos han sido altos, o poco favorables, a la medida en que ha generado resultados relacionados al aumento de las desigualdades sociales y en la calidad de vida para la gente que no tiene la posibilidad de calificarse para ocupar los cargos directivos en estos emprendimentos. De este modo, si por un lado los objetivos que fundamentan una política y los compromisos firmados en conferencias internacionales sobre la sostenibilidad, como la Eco 92 y Rio 2010, y las directrices especificas de la sostenibilidad del turismo, se reproducen en los discursos políticos, por otro lado los efectos en la realidad son distintos.

En esta realidad se encuentra Litibú y Vidanta Nuevo Vallarta, este último cuenta con el reconocimiento Earth Check y Distintivo S, lo que parece una paradoja cuando se coloca en la amplia óptica de las implicaciones que genera el desarrollo turístico en la zona de influencia.

En suma, los intereses que persigue el turismo son inherentes al sistema capitalista y se contraponen a los que persigue el desarrollo sustentable, por lo que los avances en materia de sustentabilidad son irregulares generando desequilibrios en las dimensiones que abarca (económico, social, ambiental) $y$, por tanto, se convierte en un proyecto utópico frente a los objetivos planteados por la Organización Mundial del Turismo de cara al año 2030.

\section{REFERENCIAS}

CÁRDENAS GÓMEZ, E. P. Chiapanaecos en la zona metropolitana de Puerto Vallarta. Puerto Vallarta: Universidad de Guadalajara, 2014.

CASTRO, U. Estructuras Regionales Emergentes y Desarrollo Turístico Sustentable: La región Costa Sur, Nayarit, México. Tesis Doctoral, 2010.

CAVALCANTI, P. A. B. Um Olhar Crítico sobre o Conselho Nacional de Turismo: Articulação do Setor, Legitimidade e Auto-Interesse na Construção das Políticas Públicas. 143 f. Dissertação (Mestrado) Escola de Administração de Empresas de São Paulo. São Paulo: SP, 2006.

CÉSAR DACHARY, A. El Turismo: un modelo de desarrollo. Revista Latinoamericana de Turismologia Rlat. V. 1, n. 1, 2015, pp. 16-26.

CÉSAR DACHARY, A.; ARNAIZ BURNE, S. M. Territorio $y$ Turismo. Puerto Vallarta: Universidad de Guadalajara, 2006.

CÉSAR DACHARY, A.; ARNAIZ BURNE, S. M. Territorios Globalizados del Turismo Rural. Puerto Vallarta: Universidad de Guadalajara.

CHÁVEZ, E. S.; OSORIO, J. A. Turismo y Sustentabilidad: de la teoría a la práctica en Cuba. Cuadernos de Turismo, n.17, Enero - Junio, 2012.
DÍAZ ORUETA, F. El impacto de los megaproyectos en las ciudades españolas. Hacia una agenda de investigación. Estudios Demográficos y Urbanos, p.193-218, 2009.

FIDEICOMISO DE BAHÍA DE BANDERAS. (2017). Página Oficial. Obtenido de FIBBA: http://fibba.gob.mx

FONDO NACIONAL DE FOMENTO AL TURISMO. (28 de febrero de 2010). FONATUR. Obtenido de Página Oficial:

http://www.fonatur.gob.mx/movil/es/comunicacion/index .asp?cve_sec $=222$

FONDO NACIONAL DE FOMENTO AL TURISMO. (ND). Transparencia. Obtenido de Libros Blancos: http://www.fonatur.gob.mx/gobmx/transparencia/Libros Blancos $/ 4 \% 20 \mathrm{CIP} \% 20$ Nayarit.pdf

FONSECA MORALES, M. A. Punta Mita en la dinámica del desarrollo turístico regional. El Periplo Sustentable, p.85-108, 2009.

GÓMEZ JARA, F. Acapulco: Despojo y Turismo. Problemas del Desarrollo. Revista Latinoamericana de Economía. UNAM, 126-147, 1974.

GRUPO VIDANTA. Página Oficial. Obtenido de http://www.grupovidanta.com/, Aceso en: 12 de septiembre de 2017.

GUARDADO, G. M. Turismo, globalización y desarrollo local: Puerto Vallarta y los retos del porvenir. Estudios Demográficos y Urbanos, El Colegio de México, A.C., vol. 24, núm. 1, enero-abril, 2009, pp. 219-247

INSTITUTO NACIONAL DE ESTADISSTICA Y GEOGRAFÍA. Página oficial, 2010. Obtenido de INEGI: http://www.beta.inegi.org.mx/app/areasgeograficas/?ag $=18 \#$

INSTITUTO NACIONAL PARA EL FEDERALISMO Y EL DESARROLLO MUNICIPAL. (2017). Página Oficial. Obtenido de INAFED: https://www.gob.mx/inafed

LINARES, H. L.; GARRIDO, G. M. Del desarrollo turístico sostenible al desarrollo local. Su comportamiento complejo. Revista de Turismo y Patrimonio Cultural Pasos, n.12, v.2, p.453-466, 2014.

LOPEZ, M.P.V.; GODOI, C.K. Procedimentos Metodológicos Encontrados no Turismo Sustentável. En XI Seminário da Associação Nacional Pesquisa e Pós-Graduação em Turismo. Universidade Federal do Ceará, 2014.

MACCANNELL, D. El Turista: una nueva teoría de la clase ociosa. Barcelona. 2003.

MUNTEAL, O.; SIMONET, A. C. Sustentabilidade da política de turismo no brasil e desenvolvimento do ecoturismo. XXII Congresso Pan Americano de Escolas de Hotelaria, Gastronomia e Turismo, 2012.

NARVÁEZ, J. (09 de marzo de 2009). Fracasó el centro turístico de Litibú, Nayarit, señalan ONG. La Jornada, versión en línea, pág. 37.

ORGANIZACIÓN MUNDIAL DEL TURISMO. Página Oficial, 2017. Obtenido de OMT: http://www2.unwto.org/es

ORGANIZAÇÃO MUNDIAL DE TURISMO - OMT. UNWTO Annual Report 2012. Spain. Disponible en: http://www2.unwto.org/publication/unwto-annual-report2012. Acedido en 31 de jan. 2014.

RABINOVICl, A. Ambientalismo, Organizações Não Governamentais e a Busca pela Sustentabilidade no Turismo. Turismo em Análise, n.22, v.1, 2001. 
RAMÍREZ TAMAYO, Z. (17 de mayo de 2016). La fantasía de 1,300 mdd que Vidanta llevará a Nayarit. Forbes.

RIVERA GONZÁLEZ, L. C.; CEBALLOS CHÁVEZ, L. A.; ARNAIZ BURNE, S. M. Migración y calidad de vida en Las Jarretaderas, municipio de Bahía de Banderas, Nayarit. In: ARNAIZ BURNE, S. M.; JUÁREZ MANCILLA, J. Desarrollo, Crisis y Turismo. Puerto Vallarta: Universidad de Guadalajara, p. 436 - 452, 2015.

RIVERA NAYARIT. Página Oficial. (20 de Septiembre de 2017). Obtenido de http://www.rivieranayarit.com.mx/iberostar_playa_mita_ nayarit

RUIZ OLABUÉNAGA, J.I. E ISPIZUA, M. A. La decodificación de la vida cotidiana: Métodos de la investigación cualitativa, Universidad de Deusto, Bilbao, 1989.

SALVATIERRA, N. M. La insustentabilidad del turismo. In: ALEJANDRE, J. A.; VALENZUELA, I. C. Desarrollo insostenible: gobernanza, agua y turismo. Universidad de Guadalajara, UCLA Program on México, PROFMEX-WORLD, Juan Pablos Editor, 2011.

SANTO, J. (27 de marzo de 2016). Vidanta busca expulsar a pescadores y palaperos de Boca de Tomates, Jalisco. La Jornada, versión en línea, pág. 19.
SANTO, J. (09 de Enero de 2017). Temen despojo de tierras para proyecto del Cirque du Soleil en Nuevo Vallarta. La Jornada. Versión en línea., pág. 27.

SOARES, E. B. S.\& EMMENDOERFER, M. L. Por um turismo sustentável? Uma análise à luz das abordagens de desenvolvimento no planejamento público do turismo em Minas Gerais (2007-2010). Revista Anais Brasileiros de Estudos Turísticos IABET, Juiz de Fora, v.1, n.2, p. 15-28, jul./dez. 2011.

VALDIVIA GARCÍA, J. Á. (28 de Septiembre de 2017). Percepción social en Higuera Blanca en torno a Litibú. (S. Zepeda, Entrevistador)

VALLE, A. (17 de mayo de 2016). Expansión. Obtenido de http://expansion.mx/empresas/2016/05/16/grupovidanta-el-malabarista-del-turismo-que-hoy-es-ungigante-de-10-500-mdp

VERA, F.; LÓPEZ, F.; MARCHENA, M.; ANTÓN, S. Análisis Territorial del Turismo y Planificación de Destinos Turísticos. Valencia: Tirant Humanidades, 2013.

WALLERSTEIN, I. Abrir las Ciencias Sociales. Comisión Gulbenkian para la reestructuración de las ciencias sociales. México: Siglo XXI, 2013.

World Tourism Organization - UNWTO \& United Nations Development Programme - UNDP Tourism and the Sustainable Development Goals - Journey to 2030, UNWTO, Madrid, 2017.

Processo Editorial / Editorial Process

Editor Chefe/Editor-in-chief: PhD Thiago D. Pimentel (UFJF).

Recebido em 07 de dezembro de 2017; aceito em 31 de Janeiro de 2018; publicado online em 28 de Fevereiro de 2018.

Received on December 07, 2017; accepted on January 31, 2018, published online on February 28, 2017.

Artigo original / Original article. Seção revisada por pares / Double bind review section. 